\title{
Structured propositions and trivial composition
}

\author{
Bryan Pickel ${ }^{1}$
}

Received: 25 October 2017 / Accepted: 11 June 2018 / Published online: 13 July 2018 (c) The Author(s) 2018

\begin{abstract}
Structured propositions are often invoked to explain why intensionally equivalent sentences do not substitute salva veritate into attitude ascriptions. As the semantics is standardly developed-for example, in Salmon (Frege's puzzle. Ridgeview, Atascadero, CA, 1986), Soames (Philos Top 15(1):47-87, 1987) and King (Noûs 29(4):516-535, 1995), the semantic value of a complex expression is an ordered complex consisting of the semantic values of its components. Such views, however, trivialize semantic composition since they do not allow for independent constraints on the meaning of complexes. Trivializing semantic composition risks "trivializing semantics" (King and Stanley, in: Szabó (ed) Semantics versus pragmatics, Oxford University Press, Oxford, 2005). Yet, proponents of structured propositions suggest a route to reimpose substantive compositionality. While the mapping from a sentence to the structured proposition it expresses is trivially compositional, there is a non-trivial mapping from the structured proposition to its truth-value. This non-trivial level of semantic composition allows the meanings assigned to complex expressions to impose substantive constraints on the meanings of the simple ones. I first articulate the basic desiderata: an account of structured propositions capable of explaining attitude attributions and of delivering a non-trivially compositional semantics. I then show that the two-stage semantics proposed by standard proponents of structured propositions does not satisfy these joint goals.
\end{abstract}

Keywords Semantics $\cdot$ Compositionality $\cdot$ Structured propositions

Compositionality is the thesis that the meaning of a complex expression is a function of the meanings of its constituents and their mode of combination. The thesis is a fixed point in contemporary formal semantics, meant to provide discipline and structure to semantic theorizing. It is also meant to explain how speakers can understand infinitely many complex expressions in terms of their grasp of finitely many basic expressions. However, compositionality alone cannot play these regulatory and explanatory roles.

\footnotetext{
$凶$ Bryan Pickel

bryan.pickel@ed.ac.uk

1 University of Edinburgh, Edinburgh, UK
} 
Horwich (1997) has shown that the thesis can be trivialized by treating the semantic value of a complex expression as a complex containing the semantic values of all of its constituents expressions and their mode of combination. On this type of semantics, the assignment of semantic values of complex expressions imposes no interesting constraints on the assignment of semantic values to basic expressions.

This is a problem for standard proponents of structured propositions in philosophy. Structured propositions are meant to explain why sentences with the same possibleworlds intension need not substitute salva veritate in attitude ascriptions and other very fine grained contexts. Standard proponents say that the structured proposition expressed by a sentence is a complex containing the semantic values of its constituent expressions. So the sentence (1) 'Socrates sits' will express a structured proposition which contains the semantic values of 'Socrates' and 'sits' as constituents. These semantic values might be the man Socrates himself and the property of sitting (respectively), or they might be representations of these entities. An intensionally equivalent sentence such as (2) 'Socrates sits and is distinct from Plato' has additional constituents and so expresses a different structured proposition. The vast majority of contemporary advocates of structured propositions-prominently including Salmon (1986), Soames (1987) and King (1995)_advocate a semantic theory of this kind.

The worry is that a semantic theory having roughly this shape is exactly the kind of trivial semantic theory that Horwich described. King and Stanley (2005, p. 123) likewise concede that this sort of semantic composition rule (which King himself endorses) is "trivial". This triviality threatens to result in "trivializing semantics". But King and Stanley (2005) suggest that while the mapping from sentences to structured propositions is as trivial as Horwich's semantics, there is still semantics to be done. They propose a two-stage semantics. ${ }^{1}$ And the second stage of semantics imposes substantive constraints. ${ }^{2}$ Namely, one still needs a non-trivial composition rule determining a proposition's truth-value as a result of its constituent structure. So the standard proponents of structured propositions_-including again Salmon (1986), Soames (1987), and King (1995)—offer a second mapping (or level of semantic composition) which takes one from a structured proposition to its truth-value. Because of this non-trivial level of semantic composition, the meanings assigned to complex expressions still impose substantive constraints on the meanings of the simple ones.

In this paper, I first articulate the basic desiderata: an account of structured propositions capable of explaining attitude attributions and of delivering a non-trivially compositional semantics. I then show that the two-stage semantics does not satisfy these joint goals. In particular, by introducing a substantive composition rule mapping structured propositions to truth-values, one is no longer able to explain why intensionally equivalent sentences such as such as (1) and (2) do not substitute salva veritate in attitude ascriptions. This implies that the two-stage semantics introduced by King and Stanley and others restores substantive compositionally only at the cost of undermining the reason for positing structured propositions in the first place. I conclude that proponents of structured propositions should meet the challenge head on: the mapping from sentences to structured propositions should be non-trivial.

\footnotetext{
${ }^{1}$ This is not to be confused with the two-stage semantics characterized in Almog (1986), p. 219.

${ }^{2}$ See also Johnson (2015).
} 


\section{Structured and unstructured propositions}

Standard formal semanticists treat the meaning of a sentence as an intension, a function from points of evaluation to truth-values. It follows that logically or necessarily equivalent sentences express the same proposition. Sentences (1) and (2) have the same intension (because it is necessary that Socrates is distinct from Plato). Thus, they express the same proposition.

(1) Socrates sits.

(2) Socrates sits and is distinct from Plato.

Philosophers have long been uncomfortable with this model. A typical rebuttal begins with the observation that sentences such as (3) and (4) differ both in meaning and in truth conditions despite differing only by the substitution of sentences with the same truth conditions.

(3) Sam believes that Socrates sits.

(4) Sam believes that Socrates sits and is distinct from Plato.

The worry arises if one assumes BASIC COMPOSITIONALITY (Pagin and Westerstahl $2010,254) .^{3}$

BASIC COMPOSITIONALITY: If $\phi_{\alpha}$ and $\phi_{\beta}$ differ only by the substitution of constituent $\alpha$ for constituent $\beta$ with the same semantic value, then $\phi_{\alpha}$ and $\phi_{\beta}$ have the same semantic value. ${ }^{4}$

From BASIC COMPOSITIONALITY and the fact that (3) and (4) may have different truth-values but differ only by the substitution for (1) and (2), it follows that (1) and (2) contribute something beyond their intension to the semantic values of sentences that contain them.

Friends of structured propositions propose that sentences such as (1) and (2) have structured propositions as their semantic values. A structured proposition is an arrangement of constituents, objects and properties (or their representations). Sentences (1) and (2), it is argued, express different structured propositions. So there is no obstacle to saying that (3) and (4) differ in meaning. We shall later examine how these structured propositions can lead to differences in truth-values in (3) and (4).

To incorporate this idea into a satisfactory theory, one needs to characterize how the structured propositions which are the semantic values of (1) and (2) derive from the semantic values of their sub-sentential constituents. According to a common proposal, the structured propositions expressed by (1) and (2) are composed of the semantic values of their sub-sentential constituents and the arrangement of these semantic values parallels the arrangement of the constituents of these sentences. Let $\llbracket . \rrbracket_{1}$ be the mapping from an expression to its semantic value. I assume (as is standard) that all complex

\footnotetext{
3 This thesis is also known as STRONG COMPOSITIONALITY (Larson and Segal 1995, §3.2.1).

${ }^{4}$ For simplicity, I offer only the substitutional version of basic compositionality rather than the functional version. This latter version says that for any syntactic construction rule $R$, there is a function $f$ such that for any expression $E$ which results from applying $R$ to expressions $\alpha_{1}, \ldots, \alpha_{n}$, the semantic value of $E$ is the result of applying $f$ to the semantic values of $\alpha_{1}, \ldots, \alpha_{n}$. Pagin and Westståhl (2010, p. 254) show that under minimal assumptions the substitutional and functional versions of basic compositionality are equivalent.
} 
expressions result from merging two immediate syntactic constituents. ${ }^{5}$ The proposed semantic composition rule can then be represented as follows, using solid lines to represent the relation between a complex expression and its immediate components and dashed lines as a placeholder for whatever the relation is between the semantic value of an expression and the constituents of this value.

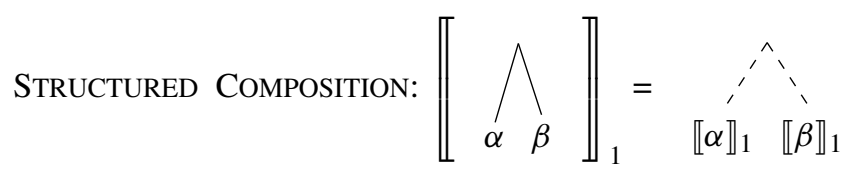

Given STRUCTURED COMPOSITION, structured propositions typically recapitulate the structure of the sentences that express them. Since (1) and (2) have different sentential structures, they contribute different structured propositions as their semantic values to sentences that contain them.

Let's call this the MIRRORING THESIS.

MIRRORING THESIS: The semantic value of a sentence is a proposition composed of the semantic values of the sentence's constituents arranged in a structure which mirrors the structure of the sentence.

Advocates of structured propositions sometimes attempt to define their view in terms of the mirroring thesis. ${ }^{6}$ An important claim of this paper is that the mirroring thesis should be rejected in order to impose substantive compositionality. Because I am concerned with natural language semantics, I focus on theories on which the semantic value of a proposition mirrors the structure of the natural language sentence that expresses it. However, many of the points made will apply to those who hold that the mirroring thesis applies only to sentences of a formal language so long as there is a systematic and explanatory translation procedure from natural language sentences into sentences of the formal language. ${ }^{7}$

\section{Trivializing semantic composition}

Proponents of STRUCTURED COMPOSITION depart from standard semantics not only by rejecting intensionalism but also by rejecting views about how the meanings of the simple expressions compose to determine the meaning of a complex expression. BASIC COMPOSITIONALITY is a rather weak requirement. Given STRUCTURED COMPOSITION, semantic composition yields an output that mirrors the syntactic structure of the input sentence. As Horwich (1997, p. 509) argued, in the absence of further requirements:

\footnotetext{
5 Nothing I say turns on whether there are other syntactic formation rules.

6 This idea is most directly on display in King (1995). However, very similar ideas are presented in Salmon (1986), Soames (1987, 2010, p. 112), Hanks (2011, p. 11ff) and Keller and Keller (2013, p. 1). There are a few exceptions to this, mostly to do with variable binding. See King (1995, footnote 22) and Pickel and Rabern (forthcoming, §1). At any rate, the point remains that syntactic structure is preserved and, as King and Stanley (2005, footnote 29) say, "the more sentence structure is preserved in proposition structure, the less composition occurs in mapping from sentence to proposition".

7 See discussion in Keller (2017).
} 
compositionality imposes no constraint on how the meaning properties of the primitives are constituted. ${ }^{8}$

There are two senses in which STRUCTURED COMPOSITION may be said to be a trivial compositional rule.

First, as it is developed in Horwich, StRUCTURED COMPOSITION is not paired with any independent constraints on the meanings of complex expressions. Reacting to Horwich, Dever argues that compositionality is a substantive constraint only if combined with other principles containing the meaning of complex expressions.

Given complete freedom to set the meanings of complexes, those meanings can always be made functions of any meanings of atomic expressions. Complete freedom to tinker at the top, then, gives a clear case in which compositionality places no constraint on meanings as before, compositionality is a tug-of-war between the meanings of parts and the meanings of complexes, and if one side lets go the rope, the war is easily won. (Dever 2006, §26.2.1)

In other words, BASIC COMPOSITIONALITY is not a substantive requirement unless we have some independent views about the semantic values of both primitive and complex expressions. The problem is that given STRUCTURED COMPOSITION, the only constraint on the meanings of the complex expressions is that they are as fine grained as the meanings of their constituents and their mode of combination.

The most obvious way to impose a substantive requirement on the meanings of complex expressions would be to hold that some sentences are synonymous and to see whether the semantic theory can assign them the same semantic value. One typical proposal is that active and passive sentences are synonymous. Thus, one might hold that $(5 \mathrm{a})$ and $(5 \mathrm{~b})$ are synonymous.

(5) (a) Annabel watches Benjamin.

(b) Benjamin is watched by Annabel.

But as Collins $(2014, \S 3)$ has pointed out, semantic composition rules such as STRUCTURED COMPOSITION do not assign these sentences the same semantic value. In the fashion typical of a proponent of STRUCTURED COMPOSITION, King (2013, p. 767) agrees that his theory does not assign the same proposition to (5a) and (5b), but argues that he should not be held to pre-theoretic constraints on which sentences say the same thing. Insofar as he is rejecting this extra-theoretic constraint on the semantic values of complex expressions, King's view of the mapping from sentences to propositions slips towards triviality.

Analogously, one might think that the sequence of sentences in (6) say the same thing, as is suggested by Kripke (2005, p. 1025, footnote 45).

(6) (a) That is green.

(b) That is such that it is green.

(c) That is such that it is such that it is green.

8 The complaint is also articulated by Johnson (2015) and King and Stanley (2005). 
But standard propositionalist semantic theories assign these sentences to different structured propositions. ${ }^{9}$

Just as there are pre-theoretic constraints making it desirable to say that two sentences in the same language can be synonymous, there are other pre-theoretic constraints making it desirable to say that two sentences in different languages may be synonymous. In particular, one would like to say that sentences in different languages may be synonyms even if the structure of language requires the sentences to have different structures. For example, the sentences (7a) of English and (7b) of Spanish would standardly be taken to be translations (and thus synonyms) despite the difference in structure.

(7) (a) Benjamin is interested in biology.

(b) A Benjamin le interesa la biología.

But STRUCTURED COMPOSITION does not deliver this result. ${ }^{10}$ In the spirit of a standard structured propositionalist, King (2013) once again responds to these objections only by arguing that although it might look plausible that they express the same proposition, in fact they do not. ${ }^{11}$

The worry here is not the standard criticism that structured propositions are too fine grained. Rather, the worry is that the mapping from sentences to structured propositions is trivial in the sense that there seem to be few to no independent constraints on the synonymy of composite expressions generated by this mapping. If semantics is meant to be genuinely explanatory, then a semantic theory should impose constraints on the semantics of composites. These constraints should lend themselves to potentially falsifiable predictions. If a semantics automatically distinguishes composite expressions with different structures or whose corresponding parts differ in meaning, then there is far less risk that the theory will be subject to these potential counterexamples.

This approach to semantics stands in opposition to a proposal by Jespersen (2015, p. 249) that a semantic theory should over-generate differences among propositions.

[A]s a general methodological maxim, it is safer to possibly overgenerate propositions. Then we always have enough of them handy should we need them.

\footnotetext{
9 Salmon (2010, p. 456) has a somewhat nuanced response to Kripke's worry. But elsewhere, Salmon (2006, p. 371, especially footnote 4) defends this distinction. King and Soames have a similar exchange about this issue in King et al. (2014, pp. 130-131, 238). The issue is extensively discussed in Jespersen (2015).

10 For example, Collins (2014) criticizes King for failing to deliver the relevant synonymies. King (2013) also cites versions of the objection arising in personal communication from Ofra Magidor and John Burgess.

11 It is worth noting that King (2016) has subsequently changed his view about the mapping from a sentence to a structured proposition. The major change is that King now regards some syntactic combination as idle. On King's revised view, active and passive pairs such as (5a) and (5b) effectively agree in all syntactic structure figuring in semantic processing. They differ only in what King (ibid, 24) regards as semantically inactive nodes such as the preposition 'by' and in their surface structure. (This requires King to take a stand on the semantic analysis of so called 'vacuous' words. He cannot, for example, accept a semantic analysis of 'by' as an identity function (Heim and Kratzer 1998, §4.1), since that would require the result of merging 'by' with 'Annabel' as determined by saturation, which King regards as semantically significant.) While this proposal helps with the examples in (5) and arguably in (7), it does not extend very widely. As King freely admits (ibid, 25), 'John solved the problem' and 'it was John who solved the problem' will still have different semantic values as will the examples in (6).
} 
Presumably, Jespersen means that if we lay down in advance that sentences $S$ and $S^{*}$ express different propositions, we will then be able to accommodate a novel observed difference. For instance, it might be found that there is a construction $\Sigma($.) such that the observational data support $\llbracket \Sigma(S) \rrbracket \neq \llbracket \Sigma\left(S^{*}\right) \rrbracket$. This data will be easier to accommodate if we already have that $\llbracket S \rrbracket \neq \llbracket S^{*} \rrbracket$. The more differences posited in the semantic values of complex expressions, the less risk there is that the semantic framework will be falsified (or forced to take unwelcome commitments) by newly observed constructions.

So Jespersen's more profligate semanticists will have an easier time describing novel phenomena using their existing framework. But this very facility in describing any novel construction using their existing framework means that the profligate semanticist is less able to make potentially falsifiable predictions about new cases. A semanticist who has explanatory-as opposed to merely descriptive ambitions-will be frustrated. A semantic theory that can easily accommodate any observed distribution of novel cases simply is making fewer potentially falsifiable predictions about novel cases. $^{12}$

The second way in which STRUCTURED COMPOSITION may be said to be trivial is that the composition rule seems capable of applying regardless of the types of entities assigned to the basic semantic values. ${ }^{13}$ Standard semantics since Frege makes use of a function and argument composition rule, where the semantic value of a complex expression is the result of applying (or "saturating") the function denoted by one component to the argument denoted by the other component. King and Stanley speak of this composition rule as being "intuitively correct", but it is easier to defend by citing the work it does in standard semantics. For instance, Dever (2012: p. 69) and Jacobson (1996, p. 90) both point out that in Montague semantics (which makes use of a typed function and argument composition rule), the meaning of a complex expression and one of its components impose tight constraints on the meanings of its other components. STRUCTURED COMPOSITION imposes no corresponding constraints on the semantic values of the primitive expressions. In this way, one might view it as trivializing semantic composition.

\section{Two-stage semantics}

On the structured proposition view outlined so far, "no significant composition of semantic contents of the elements of a sentence occurs in the mapping from sentence to [structured proposition]" (King and Stanley 2005, p. 134, note 29). Because of the structural isomorphism between a sentence and the structured proposition it expresses, one might be tempted to think of the result of applying $\llbracket . \rrbracket_{1}$ to a sentence $S$ as a

\footnotetext{
12 This is not to say that Jespersen's maxim is wrong. Rather, it is limited in application. If one's goal is a maximally flexible descriptive framework, then Jespersen is correct. However, if one wants an empirically constrained, explanatory semantic theory, then the semanticist should aim for falsifiable predictions.

13 I take this to be what King and Stanley mean when they criticize views which deliver STRUCTURED COMPOSITION as follows: "[O]ne does not compose the characters in accord with the intuitively correct composition rules governing its syntactic structure. For example, one does not 'saturate' [the semantic value of one component expression with the semantic value of the other]" (King and Stanley 2005, p. 123).
} 
"translation" of the sentence into another structured representation system (Lewis 1970, pp. 18-19). As a result, standard structured propositionalists say that the trivial mapping $\llbracket . \rrbracket_{1}$ does not exhaust semantic processing. This mapping merely outputs the structured proposition associated with a sentence. The semantic theory also needs to specify the sentence's truth conditions in terms of the proposition it expresses. For those who think of a proposition as itself a representational entity, it is then natural to think of this further stage as genuinely semantic processing of the structured proposition. I will represent this mapping from structured propositions onto their truth conditional contributions as $\llbracket \cdot \rrbracket_{2}$.

Insofar as a semantic theory assigns a truth condition to each sentence of a language, it imposes some falsifiable empirical predictions. In particular, we do judge an individual sentence to be true or false in a circumstance. However, for a semantic theory to be truly substantive, there must be tight constraints on the semantic contribution a sentence can make to the truth conditions of a sentence that contains it. There's no use in a semantic theory that assigns truth conditions to sentences on a case-by-case basis. At a minimum, the mapping ought to be compositional. But more than that, the mapping should be non-trivial. For instance, if the values of any two atomic expressions differ and the semantic values of any two complexes preserve information about the arrangement and semantic values of their constituents, then any theory assigning truth conditions to complex expressions will satisfy basic compositionality. A theory that assigns a rich enough semantic value to every sentence can accommodate the sentence's contribution to the truth conditions of any sentence that contains it.

The type of semantic theory under consideration assigns a truth condition to each sentence by way of assigning the sentence to a proposition and then assigning the proposition to a truth condition. We have seen the sense in which the mapping $\llbracket \cdot \rrbracket_{1}$ from sentences to propositions is trivial. Proponents of structured propositions can then see the second mapping $\llbracket \cdot \rrbracket_{2}$ as reimposing substantive constraints on compositionality. ${ }^{14}$ King and Stanley (2005, p. 134, footnote 29) describe this two-stage process as follows.

[T]he structures of propositions are identical to the structures of sentences expressing them (in a context). Thus, the semantic contents (in the context) of the lexical items in the sentence are not composed in any significant way in the mapping to the proposition: the proposition is literally the concatenation of these semantic contents, and is structurally identical to the sentence expressing it. By contrast, in the mapping from propositions to truth-values, there is significant composing of the constituents of the proposition (i.e. the semantic contents of the lexical items in the sentence expressing the proposition) to yield a truthvalue. ...[O]n any theory of structured propositions that preserves most of the structure of a sentence in the structure of the proposition, most of the significant composition of semantic contents occurs in the mapping from propositions to truth-values.

The mapping $\llbracket \cdot \rrbracket_{2}$ imposes substantive conditions on composition in two ways. First, it provides an independent check on the semantic values assigned to complex expressions. The truth conditions of a sentence $S$ can be given by composing the trivial

14 See King and Stanley (2005, p. 134) and Johnson (2015, §4a). 
mapping $\llbracket . \rrbracket_{1}$ of a sentence $S$ onto a structured content with the non-trivial mapping $\llbracket . \rrbracket_{2}$ of a structured content onto its truth conditions, to yield the function $\llbracket \llbracket . \rrbracket_{1} \rrbracket_{2}$. Moreover, given the assumption that syntactic structure mirrors the structure of content, the truth conditional contribution of an expression $E$ will be $\llbracket \llbracket E \rrbracket_{1} \rrbracket_{2}$. There are independent constraints governing when sentences $S$ and $S^{*}$ have the same truth conditions. If they do, then $\llbracket \llbracket S \rrbracket_{1} \rrbracket_{2}=\llbracket \llbracket S^{*} \rrbracket_{1} \rrbracket_{2}$. So we have an independent check on the semantic theory.

Second, the mapping $\llbracket . \rrbracket_{2}$ can impose typing constraints on the constituents of the structured proposition. When applied to the structured content of a sentence, $\llbracket \cdot \rrbracket_{2}$ outputs the content's truth conditions in terms of a non-trivial function $f$ applied to the truth conditional contributions of the content's constituents.

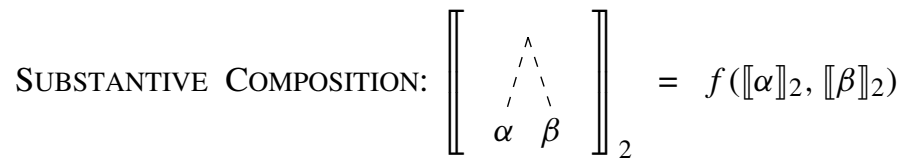

The function $f$ may be sensitive to the types assigned to its arguments. So, the twostage semantics addresses the complaint that STRUCTURED COMPOSITION does not suitably constrain the types assigned to the primitive expressions. Thus, in deriving the truth conditions of a sentence via a non-trivial compositional rule, it is hoped that compositionality can again significantly constrain semantic theorizing. ${ }^{15}$

\section{A slingshot argument}

While this strategy introduces non-trivial compositionality in the semantic theory, it undermines the core motivation for positing structured propositions. In particular, structured propositions were posited to distinguish the truth conditions of attitude ascriptions such as (3) and (4).

(3) Sam believes that Socrates sits.

(4) Sam believes that Socrates sits and is distinct from Plato.

Sentences (3) and (4) differ only by the substitution of (1) 'Socrates sits' and (2) 'Socrates sits and is distinct from Plato', which have the same truth conditions. That is, $\llbracket \llbracket(1) \rrbracket_{1} \rrbracket_{2}=\llbracket \llbracket(2) \rrbracket_{1} \rrbracket_{2}$.

The proposal to restore substantive compositionality in the mapping from structured propositions to truth conditions along the lines of SUBSTANTIVE COMPOSITION yields the result that (3) and (4) agree in truth conditions. Since (1) and (2) contribute only $\llbracket \llbracket(1) \rrbracket_{1} \rrbracket_{2}$ and $\llbracket \llbracket(2) \rrbracket_{1} \rrbracket_{2}$ respectively to the truth conditions of sentences that contain them, it follows that $\llbracket \llbracket(3) \rrbracket_{1} \rrbracket_{2}=\llbracket \llbracket(4) \rrbracket_{1} \rrbracket_{2}$. That is:

$\llbracket \llbracket$ Sam believes that Socrates sits $\rrbracket_{1} \rrbracket_{2}=\llbracket \llbracket$ Sam believes that Socrates sits and is distinct from Plato $\rrbracket_{1} \rrbracket_{2}$.

15 Pickel (2017, p. 4, footnote 5) briefly considers this two-stage strategy, expressing skepticism about whether the second stage is genuine semantics. By way of contrast, this paper takes seriously the view of King and Stanley that this second stage of semantics is semantics. 
This can be seen from the following argument for the conclusion that anyone who embraces SUBSTANTIVE COMPOSITION should identify the truth conditions of (3) and (4).

(A)

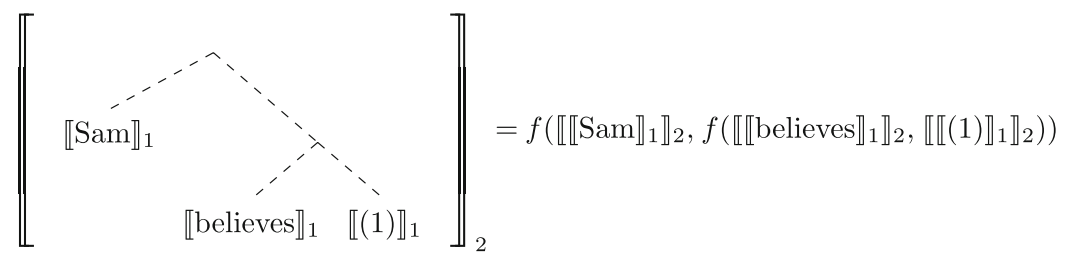

(B) $f\left(\llbracket \llbracket\right.$ Sam $\rrbracket_{1} \rrbracket_{2}, f\left(\llbracket \llbracket\right.$ believes $\left.\left.\rrbracket_{1} \rrbracket_{2}, \llbracket \llbracket(1) \rrbracket_{1} \rrbracket_{2}\right)\right)=f\left(\llbracket \llbracket \operatorname{Sam} \rrbracket_{1} \rrbracket_{2}, f\left(\llbracket \llbracket\right.\right.$ believes $\left.\left.\rrbracket_{1} \rrbracket_{2}, \llbracket \llbracket(2) \rrbracket_{1} \rrbracket_{2}\right)\right)$

(C)

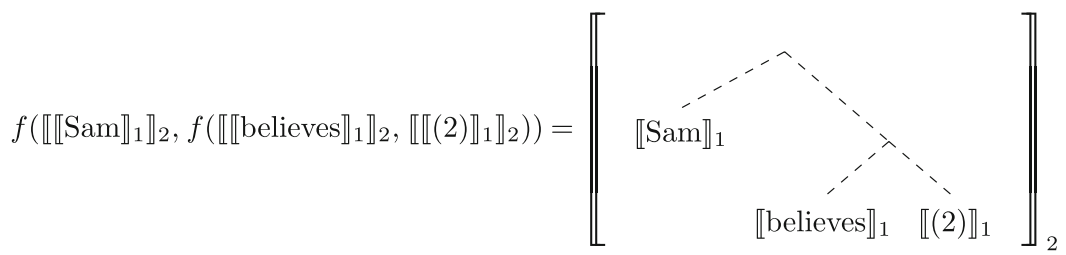

(A) and (C) are simply instances of Substantive Composition. (B) is an instance of the indiscernibility of identicals. It follows that (3) and (4) have the same truth conditions. Avoiding this consequences was the whole point of positing structured propositions as the semantic values of sentences. So the proponent of structured propositions cannot introduce substantive compositionality in the mapping from structured propositions to truth conditions without undermining her theory. ${ }^{16}$

\section{Responses}

The argument above relies on three premises about how the mapping $\llbracket . \rrbracket_{2}$ applies to structured propositions.

INTENSIONALISM: The truth conditional contribution $\llbracket p \rrbracket_{2}$ of a proposition $p$ is the intension of $p$. So propositions with the same truth conditions make the same contribution to the truth conditions of sentences that contain them,

BASIC COMPOSITIONALITY: The mapping from propositions to their truth conditional contribution $\llbracket \cdot \rrbracket_{2}$ satisfies BASIC COMPOSITIONALITY, and

SUBSTITUTION: Propositional attitude ascriptions of the form $\ulcorner\alpha$ believes that $\phi\urcorner$ and $\ulcorner\alpha$ believes that $\psi\urcorner$ express propositions which differ only by the substitution of the propositions expressed by $\phi$ and $\psi$.

The argument could be resisted by denying any of these premises. But I shall now argue that is unwise for the standard structured propositionalist who endorses STRUCTURED COMPOSITION to reject any of them.

16 Davidson (1967) is a classic statement of a slingshot argument that develops themes in Gödel (1944) and Quine (1953). As far as I am aware, the label "slingshot argument" derives from Barwise and Perry (1981). The particular slingshot argument offered here is independent of these other slingshots arguments. 


\subsection{Denying intensionalism}

One might distinguish the possible worlds truth conditions of a structured proposition from the contribution that proposition makes to the truth conditions of propositions that contain it. This could be accomplished in one of two ways. One could say that the proposition contributes itself to the truth conditions of propositions that contain it. In this case, the mapping from structured propositions to their truth conditional contribution would be trivial. In particular, since any sentence $S$ can be embedded in a belief ascription, the proposition $p$ expressed by $S$ contributes itself to the truth conditions of propositions that contain it. That is, $\llbracket p \rrbracket_{2}=p$ (or $\llbracket p \rrbracket_{2}$ is identical to $p$ paired with the truth conditions of $\mathrm{p}$ ). Since structured propositions are individuated in terms of their constituents, every constituent of $p$ will similarly need to contribute itself to the truth conditions of a proposition that contains $p .{ }^{17}$

Alternatively, one could say that the truth conditional contributions of propositions are entities of other kinds that are more fine grained than a set of possible worlds. The mapping $\llbracket \cdot \rrbracket_{2}$ could be a function a proposition to one of these more fine grained entities. For instance, Church (1951), Bealer (1982), Schiffer (2003) and Keller (2014) all posit fine grained, but simple entities as the objects of attitudes. One might take the truth conditional contribution of a structured proposition to be one of the simple fine grained entities. A different view says that these intermediate objects are sets of truth supporting circumstances but there are circumstances which are able to distinguish among metaphysically or even logically equivalent sentences. ${ }^{18}$

The worry with any version of this approach is that these intermediate contents can play the role fitted for structured propositions. In particular, these intermediate contents can be the values of $\llbracket \cdot \rrbracket_{1}$. There is simply no need for structured propositions in the semantic story. Moreover, the advocate of structured propositions would be prohibited from offering metaphysical arguments against these entities, since they are needed anyways by the semantics as the values of $\llbracket \cdot \rrbracket_{2}$.

\subsection{Denying basic compositionality}

A related approach would say that $\llbracket . \rrbracket_{2}$ does not satisfy BASIC COMPOSITIONALITY. For concreteness, consider two propositions $\phi_{\alpha}$ and $\phi_{\beta}$ differing by the substitution of propositional constituent $\alpha$ for propositional constituent $\beta$. Denying BASIC COMPOSITIONALITY means that it is possible to have a case where $\llbracket \phi_{\alpha} \rrbracket_{2} \neq \llbracket \phi_{\beta} \rrbracket_{2}$ but $\llbracket \alpha \rrbracket_{2}$ $=\llbracket \beta \rrbracket_{2}$. It may be that the immediate constituents contribute more than their values under $\llbracket . \rrbracket_{2}$ to the truth conditions of sentences that contain them. One way of imple-

\footnotetext{
17 Duží (2017) seemingly develops a view along these lines. On her view, the semantic value of an expression is a logical procedure, which is her notion of a structured proposition. Within a procedure, other sub-procedures occur either as executed in an extensional or intensional context or as displayed in an attitude ascription. If a procedure occurs as executed, it contributes its product. If it occurs as displayed, it contributes itself and not its product. According to Duží (2017, p. 14), "all the subconstructions of a displayed construction occur displayed as well". So a sentence retains all of the information about the semantic values of all its constituents to contribute to its semantic values in displayed contexts.

18 One might think of the semantics of Kratzer (1989), Barwise and Perry (1999), and Ripley (2012, p. $111)$ as pointing in this direction.
} 
menting this would be to say that propositions $p$ and $q$ make the same truth conditional contribution if (i) they have the same intension; (ii) they have isomorphic constituent structures; and (iii) corresponding nodes in the structures of $p$ and $q$ make the same truth conditional contribution.

Weakening compositionality is not a live option for the advocate of structured propositions who is motivated by the argument above. The argument purports to show that sentences with the same truth conditions such as (1) and (2) must have different semantic values because they embed differently in (3) and (4). But if we weaken compositionality in the way proposed, then this argument no longer applies. Even if $\llbracket(1) \rrbracket_{1}=\llbracket(2) \rrbracket_{1}$, the sentences may nevertheless make different semantic contributions to the sentences that contain them. As a result, there is no threat of identifying the truth conditions or semantic content of (3) and (4). So the argument for structured propositions above seemingly relies on BASIC COMPOSITIONALITY.

\subsection{Denying substitution}

The final option that I consider denies that $\llbracket \alpha$ believes that $\phi \rrbracket_{1}$ and $\llbracket \alpha$ believes that $\psi \rrbracket_{1}$ differ only by the substitution of $\llbracket \phi \rrbracket_{1}$ for $\llbracket \psi \rrbracket_{1}$. But this claim follows immediately from the plausible assumption that $\ulcorner\alpha$ believes that $\phi\urcorner$ and $\ulcorner\alpha$ believes that $\psi\urcorner$ differ only by the substitution of $\phi$ for $\psi$ together with the assumption of STRUCTURED COMPOSITION for $\llbracket . \rrbracket_{1}$. To deny SUBSTITUTION, a structured propositionalist would have to deny one of these components of the standard structured propositionalist semantics.

One might interpret Cresswell $(1985,2002)$ as holding that insofar as intensionally equivalent sentences don't substitute salva veritate in attitude ascriptions, they are not really syntactic constituents of the attitude ascriptions. In particular, Cresswell thought that (on one disambiguation) attitude ascriptions were exceptional constructions and so did not follow the ordinary composition procedure. For instance, in the propositional attitude ascription (3) 'Sam believes that Socrates sits', the name 'Socrates' might not combine with 'sits', but rather might first combine with 'that'. Only then does the construction 'that Socrates' combine with 'sits'. Someone advocating such a radical syntax might hold that the structured proposition expressed by $\phi$ is not a constituent of the structured proposition expressed by $\ulcorner\alpha$ believes that $\phi\urcorner$.

But, King (2007) highlights the problem with such an approach for a proponent of structured propositions.

[Advocates of Structured Propositions] think precisely that the semantics of verbs of propositional attitude require structured propositions. Their arguments in favor of structured propositions have to my knowledge always invoked this claim. ... [T] he justification for structured propositions is that they contribute to the determination of the intensions of some sentences. Indeed, the structured proposition theorist will see in this a point in favor of structured propositions: they are required to determine the intensions of some sentences. Isn't that precisely the point structured proposition theorists have been insisting on all along? (King 2007, p. 119) 
A central argument for structured propositions is that they resolve a puzzle concerning why $\phi$ cannot be substituted salva veritate for intensionally equivalent $\psi$ in $\ulcorner\alpha$ believes that $\phi\urcorner$. So, if the advocate of structured propositions rejects the standard syntax for attitude ascriptions by denying that $\phi$ is a constituent of the structured proposition expressed by $\ulcorner\alpha$ believes that $\phi\urcorner$, then they risk losing a central argument for structured propositions in the first place.

The alternative is to deny that STRUCTURED COMPOSITION holds for $\llbracket . \rrbracket_{1}$. Insofar as the aim of this paper is to undermine STRUCTURED COMPOSITION (and with it the MiRRORING THESIS), this is a welcome result. Perhaps though, the damage could be contained, since the proponent of STRUCTURED COMPOSITION might claim that attitude ascriptions are anomalous. They are special exceptions to the rules of ordinary semantic processing. Some implementations of this view would go on to explain the failure of substitution of intensionally equivalent sentences in attitude ascriptions without ever needing to appeal to anything like structured propositions. So positing a special composition rule for attitude ascriptions once again seems to undermine a major motivation for positing structured propositions in the first place.

A different approach would say that the structured proposition expressed by an attitude ascription contains a constituent standing in a special relation to the structured proposition expressed by the sentence embedded in the 'that'-clause of the attitude ascription. For example, Chalmers $(2011, \S 7)$ advocates a view that generally follows STRUCTURED COMPOSITION for the mapping between a sentence and the structured proposition it expresses. However, he seemingly adds an exceptional contribution rule for 'that'-clauses. An expression of the form $\ulcorner$ that $\phi\urcorner$ express the canonical intension of the proposition expressed by $\phi$. Chalmers represents the function mapping a proposition to its canonical intension as $A$. So, his exceptional composition rule is as follows.

CHALMERS's EXCEPTIONAL COMPOSITION: $\bigwedge_{\text {that } \phi} \|_{1}=A\left(\llbracket \phi \rrbracket_{1}\right)$

Importantly, Chalmers's mapping $A$ is a bijection. So the structured proposition expressed by $\phi$ is recoverable from its mapping under $A$ and vice-versa. From this perspective, Chalmers's proposal more closely resembles strategies denying INTENSIONALISM than it does proposals denying SUBSTITUTION. ${ }^{19}$ On this understanding, Chalmers has a structured proposition contribute itself (or its canonical intension) to the truth conditions of belief ascriptions which ascribe it. So, despite a few bells and whistles, I do not believe that this type of approach avoids the threat of trivial compositionality.

19 This suspicion is confirmed when one recognizes that Chalmers could give an equivalent semantics that
preserves structured composition for attitude ascriptions as follows.

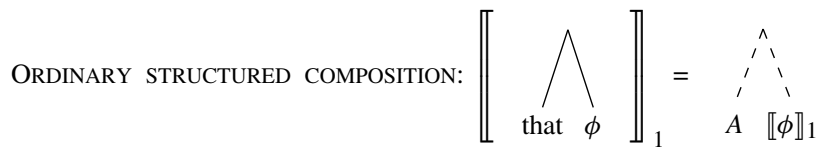




\section{A modest proposal}

In light of these considerations, one might be tempted to retreat to an unstructured conception of propositions. Yet, the situation is not so desperate. I suggest that the best solution for the advocate of structured propositions is to abandon the MIRRORING THESIS and STRUCTURED COMPOSITION. In particular, I suggest an alternative semantic theory that treats a structured proposition as the output of the function denoted by one sentential constituent to the argument denoted by the other sentential constituent. ${ }^{20}$ On this type of view, sentences with different structures - such as an active and its corresponding passive or a sentence and its standard translation-may express the same structured proposition. Thus, the proponents of structured propositions need not reject pre-theoretic checks on the assignments of semantic values to complex expressions. They can also accept typing constraints on which semantic values may combine. The mapping from sentences to structured propositions is therefore non-trivial. Because the mapping from sentences to structured propositions is already non-trivial, there is no need for the mapping from propositions to truth-values to be non-trivial. So a proposition may contribute itself to the truth conditions of a proposition that contains it without wholly trivializing semantic composition. ${ }^{21}$

Open Access This article is distributed under the terms of the Creative Commons Attribution 4.0 International License (http://creativecommons.org/licenses/by/4.0/), which permits unrestricted use, distribution, and reproduction in any medium, provided you give appropriate credit to the original author(s) and the source, provide a link to the Creative Commons license, and indicate if changes were made.

\section{References}

Almog, J. (1986). Naming without necessity. Journal of Philosophy, 83(4), 210-242.

Asher, N. (1993). Reference to abstract objects in discourse (Vol. 50). Dordrecht: Springer.

Barwise, J., \& Perry, J. (1999). Situations and attitudes. Cambridge: Cambridge University Press.

Barwise, J., \& Perry, J. (1981). Semantic innocence and uncompromising situations. Midwest Studies in Philosophy, 6(1), 387-404.

Bealer, G. (1982). Quality and concept. Oxford: Clarendon Press.

Chalmers, D. J. (2011). Propositions and attitude ascriptions: A Fregean account. Noûs, 45(4), 595-639.

Church, A. (1951). A formulation of the logic of sense and denotation. In P. Henle, H. M. Kallen, \& S. K. Langer (Eds.), Structure, method, and meaning: essays in honor of Henry M. Sheffer (pp. 3-24). New York: Liberal Arts Press.

Collins, J. (2014). Cutting it (too) fine. Philosophical Studies, 169(2), 143-172.

\footnotetext{
20 Versions of this idea can be found in Dummett (1981, p. 294), Pagin and Pelletier (2007), Elbourne (2011, pp. 104-107), and Pickel (2013, 2017). Van der Sandt (1992), Asher (1993), and Muskens (1996) develop an analogous strategy for compositionally determining the representation structure (which also serves to model the objects of the attitudes) expressed by a discourse in the context of Kamp's (1981) Discourse Representation Theory.

21 This paper was improved as a result of feedback from Geoff Georgi, Bjørn Jespersen, John Keller, Lorraine Juliano Keller, Jeff King, Brian Rabern, David Rey, Anders Schoubye, Jason Stanley, Wolfgang Schwarz, and two anonymous referees for Synthese. It also benefited from feedback from audiences at the Edinburgh Philosophy of Language Workshop and Workshop 1 of the Foundations and Methods of Natural Language Semantics at the University of Barcelona. Research related to this project was partially funded by a project FFI2016-80636-P, supported by the Spanish Government.
} 
Cresswell, M. J. (1985). Structured meanings: The semantics of propositional attitudes. Cambridge, MA: The MIT Press.

Cresswell, M. J. (2002). Why propositions have no structure. Noûs, 36, 643-662.

Davidson, D. (1967). Truth and meaning. Synthese, 17(1), 304-323.

Dever, J. (2006). Compositionality. In E. Lepore \& B. Smith (Eds.), The Oxford hand-book of philosophy of language (pp. 633-666). Oxford: Oxford UP.

Dever, J. (2012). Formal semantics. In García-Carpintero and Kölbel (pp. 47-83).

Dummett, M. (1981). Frege: Philosophy of language. Cambridge, MA: Harvard University Press.

Duží, M. (2017). If structured propositions are logical procedures then how are procedures individuated? Synthese, 19, 178.

Elbourne, P. (2011). Meaning: A slim guide to semantics. Oxford: Oxford University Press.

García-Carpintero, M., \& Kölbel, M. (Eds.). (2012). The continuum companion to the philosophy of language. London: Continuum Publishing Group.

Gödel, K. (1944). Russell's mathematical logic. In P. A. Schilpp (Ed.), The philosophy of Bertrand Russell (pp. 125-153). La Salle: Open Court.

Hanks, P. W. (2011). Structured propositions as types. Mind, 120(477), 11-52.

Heim, I., \& Kratzer, A. (1998). Semantics in generative grammar. Malden: Blackwell Publishers.

Henle, P. (1951). Structure, method, and meaning: Essays in honor of Henry M. Sheffer. New York, NY: Liberal Arts Press.

Horwich, P. (1997). The composition of meanings. Philosophical Review, 106(4), 503-532.

Jacobson, P. (1996). The syntax/semantics interface in categorial grammar. In Lappin (pp. 89-116).

Jespersen, B. (2015). Should propositions proliferate? Thought: A Journal of Philosophy, 4(4), $243-251$.

Johnson, M. (2015). Compositionality. In The internet encyclopedia of philosophy. http://www.iep.utm. edu/composit/.

Kamp, H. (1981). A theory of truth and semantic representation. In Portner and Partee (pp. 189-222).

Keller, L. J. (2014). The metaphysics of propositional constituency. Canadian Journal of Philosophy, 43(56), 655-678.

Keller, L. J. (2017). What propositional structure could not be. Synthese, 194, 5023.

Keller, L. J., \& Keller, J. A. (2013). Compositionality and structured propositions. Thought: A Journal of Philosophy, 2(4), 313-323.

King, J., \& Stanley, J. (2005). Semantics, pragmatics, and the role of semantic content. In Z. Szabó (Ed.), Semantics versus pragmatics (pp. 111-164). Oxford: Oxford University Press.

King, J. C. (1995). Structured propositions and complex predicates. Noûs, 29(4), 516-535.

King, J. C. (2007). The nature and structure of content. Oxford: Oxford University Press.

King, J. C. (2013). On fineness of grain. Philosophical Studies, 163(3), 763-781.

King, J. C. (2016). On propositions and fineness of grain (again!). Synthese, 1-25, 495.

King, J. C., Soames, S., \& Speaks, J. (2014). New thinking about propositions. Oxford: Oxford University Press.

Kratzer, A. (1989). An investigation of the lumps of thought. Linguistics and Philosophy, 12(5), 607-653.

Kripke, S. (2005). Russell's notion of scope. Mind, 114(456), 1005-1037.

Lappin, S. (1996). The handbook of contemporary semantic theory. Malden, MA: Blackwell Reference.

Larson, R., \& Segal, G. (1995). Knowledge of meaning: An introduction to semantic theory. Cambridge, MA: The MIT Press.

Lepore, E., \& Smith, B. C. (2006). The oxford handbook of the philosophy of language. Oxford: Oxford University Press.

Lewis, D. (1970). General semantics. Synthese, 22(1-2), 18-67.

Muskens, R. (1996). Combining Montague semantics and discourse representation. Linguistics and philosophy, 19(2), 143-186.

Pagin, P., \& Pelletier, F. J. (2007). Content, context and composition. In G. Preyer \& G. Peter (Eds.), Context-sensitivity and semantic minimalism: New Essay on semantics ans pragmatics (pp. 25-62). Oxford: Oxford University Press.

Pagin, P., \& Westerstahl, D. (2010). Compositionality I: Definitions and variants. Philosophy Compass, 5(3), 250-264.

Pickel, B. (2013). Russell on incomplete symbols. Philosophy Compass, 8(10), 909-923.

Pickel, B. (2017). Structured propositions in a generative grammar. Mind, fzw074. https://doi.org/10.1093/ mind/fzw074. 
Pickel, B., \& Rabern, B. (2016). The antinomy of the variable: A Tarskian resolution. Journal of Philosophy, $113,137$.

Portner, P. H., \& Partee, B. H. (2002). Formal semantics: The essential readings. Oxford: Wiley.

Preyer, G., \& Peter, G. (2007). Context-sensitivity and semantic minimalism: New essays on semantics and pragmatics. Oxford: Oxford University Press.

Quine, W. V. (1953). Three grades of modal involvment. Proceedings of the XIth International Congress of Philosophy, 14, 65-81.

Ripley, D. (2012). Structures and circumstances: Two ways to fine-grain propositions. Synthese, 189, 97118.

Salmon, N. (1986). Frege's puzzle. Atascadero, CA: Ridgeview.

Salmon, N. (2006). The resilience of illogical belief. Nô̂s, 40(2), 369-375.

Salmon, N. (2010). Lambda in sentences with designations: An ode to complex predication. Journal of Philosophy, 107(9), 445-468.

Schiffer, S. R. (2003). The things we mean. Oxford: Clarendon.

Schilpp, P. A., \& Russell, B. (1944). The philosophy of Bertrand Russell. The library of living philosophers. Evenston: Northwestern University.

Soames, S. (1987). Direct reference, propositional attitudes, and semantic content. Philosophical Topics, 15(1), 47-87.

Soames, S. (2010). Philosophy of language. Princeton, NJ: Princeton University Press.

Szabó, Z. G. (2005). Semantics versus pragmatics. Oxford: Oxford University Press.

Van der Sandt, R. A. (1992). Presupposition projection as anaphora resolution. Journal of Semantics, 9(4), 333-377. 